\title{
Intelligent Autonomous Transport Systems Design and Simulation
}

\author{
Cheng Siong Chin ${ }^{D},{ }^{1}$ Xionghu Zhong, ${ }^{2}$ Mohammad Hamdan, ${ }^{3}$ \\ Rongxin Cui $\mathbb{D}^{4},{ }^{4}$ Juan C. Cano ${ }^{1},{ }^{5}$ and Jose R. Martinez-de Dios ${ }^{6}$ \\ ${ }^{1}$ Newcastle University in Singapore, Ngee Ann Polytechnic, SIT Building, Singapore 599493 \\ ${ }^{2}$ Nanyang Technological University, 50 Nanyang Avenue, Singapore 639798 \\ ${ }^{3}$ Heriot-Watt University, Dubai Campus, Dubai, UAE \\ ${ }^{4}$ Northwestern Polytechnical University, Xi'an 710072, China \\ ${ }^{5}$ Universitat Politècnica de València, Camí de Vera, s/n, 46022 València, Spain \\ ${ }^{6}$ University of Seville, Calle San Fernando 4, 41004 Sevilla, Spain
}

Correspondence should be addressed to Cheng Siong Chin; cheng.chin@ncl.ac.uk

Received 8 February 2018; Accepted 8 February 2018; Published 11 March 2018

Copyright (C) 2018 Cheng Siong Chin et al. This is an open access article distributed under the Creative Commons Attribution License, which permits unrestricted use, distribution, and reproduction in any medium, provided the original work is properly cited.

The automation transport system is essential in increasing the communication, control, and level of autonomy of a broad range of applications in land, sea, and air. The increase in workforce age and cost has driven the technological development and adaptation of innovative system design, simulation, and application of intelligence in transport systems. The changing technological demand and operating uncertainties have resulted in new types of transportation challenges and increased demand for sensors and sensor platforms for capturing mobility analytics under uncertain operating environment. For example, various intelligent autonomous systems such as unmanned aerial and submersible vehicle for traffic survey, marine sediment transport, and vehicle accident survey are used. For land transport system, the autonomous vehicles such as Google autonomous car and Tesla are focusing on the vehicle's self-information and are developed based on the Light Detection and Ranging (LiDAR) systems, radar, and cameras. The Vehicular-to-Everything (V2X) communication technology enables cars to communicate with each other and with surrounding infrastructure access points like roadside units (RSUs). It can provide global information of the networked vehicles and has drawn extensive attention due to its broad application prospects including advanced driver assistance systems, enhanced collision avoidance, and automatic toll fee collection. In all these developments toward intelligent autonomous transport systems, there are apparently a lot of significant problems and challenges in system design and simulation that must be faced. Efficient system design and simulation using artificial intelligence techniques could help to address such challenging issues in the intelligent autonomous transport systems. In this particular issue on intelligent autonomous transport systems design and simulation, we have invited few papers that address such issues.

One paper of this special issue addresses ecodriving advisory system (EDAS) to reduce $\mathrm{CO} 2$ emissions and energy consumption by letting the continuous vehicle pass through multiple intersections with the minimum possibilities of stops. Two strategies including maximized throughput model (MTM) and smooth speed model (SSM) were designed and compared with maximized throughput model (MaxTM), open traffic light control model (OTLCM), and predictive cruise control (PCC) models. Another paper introduced a novel method for obtaining good quality paths for autonomous road vehicles in narrow environments. The approach consists of a global planner which generates a preliminary path consisting of straight and turning-in-place primitives and a local planner to make the preliminary path feasible to car-like vehicles. The approximation process with 
the proposed local planner was proven to be convergent for any preliminary global paths.

One of the papers presented a method to analyze the nonlinear dynamics and stability in lane changes on highways for tractor-semitrailer under rainy weather. The five degrees of freedom mechanical model with nonlinear tire based on vehicle test was used in the lane change simulation on low adhesion coefficient road. A feedback linearization controller combined with Active Front Steering (AFS) and DYC (Direct Yaw Control) was used to eliminate bifurcations and to improve lateral stability of tractor-semitrailer, during lane changing on the highway under rainy weather. Another paper investigated the vehicle platoon problems where the actuator saturation and absent velocity measurement were taken into consideration. A novel algorithm, where a smooth function was introduced to deal with the sharp corner of the input signals, was proposed for a group of vehicles with actuator saturation by using the consensus theory. A control strategy for the vehicle platoon with actuator saturation and absent velocity measurement was successfully designed via the adaptive control approach.

Another paper presented a unique method for designing advanced driver assistance systems (ADAS) to minimize the costs of the design phase and system implementation and, at the same time, to maximize the positive effect the system has on driver and vehicle safety. The described method was based on using a virtual prototyping tool to simulate the system performance in real life situations. The approach enabled an iterative design process that resulted in a reduction of errors with almost no prototyping and testing costs. It leads to another paper on the use of the genetic programming which was proposed to give good results in the field of tasks scheduling in the design phase. In the improved genetic algorithm, a mix of random initialization population by combining initialization machine and initialization operation with random initialization was designed for generating highquality initial population. One of the papers tried to solve the problem associated with the precise prediction of the dynamic trajectory of an autonomous vehicle. A Mivar expert system was integrated into the control system of the experimental autonomous vehicle. The system was made more flexible and efficient by the introduction of hybrid artificial intelligence with logical reasoning for an autonomous transport vehicle being involved in a collision.

One of the papers described a new rapidly exploring random tree (RRT), that is, liveness-based RRT (Li-RRT) to address autonomous vehicles motion. Different from typical RRT, an index of each node in the random searching tree named "liveness" to describe the potential effectiveness of the expanding process was used. The expected time of returning a valid path with Li-RRT was improved to verify the efficiency of the algorithm. Another paper used potential field method to navigate a three omnidirectional wheels' mobile robot to avoid obstacles. The potential field method was used to overcome the local minima problem and the goals unreachable with obstacles nearby (GNRON) problem. Also, model predictive control (MPC) was used to incorporate motion constraints to make the velocity more realistic and flexible in the mobile robot.
Another paper applied a comparative method to assess the performance of artificial neural network's (ANN) direct inverse control (DIC-ANN) with the proportional-integralderivative (PID) control system. The comparison served as an analysis tool to assess the advantages of DIC-ANN over conventional control method for an unmanned aerial vehicle (UAV) attitude controller. The DIC-ANN performed learning mechanism to overcome the limitation of PID tuning. Another paper presented a reactive path planning approach named Dubins-APF (DAPF) to solve the path planning problem for docking in an unknown environment with obstacles. The Dubins curves were combined with the designed obstacle avoidance potential field for planning a possible pathway. The robot was navigated to the docking station with proper pose via the DAPF approach.

One of the papers presented an integrated state-of-charge (SOC) estimation model and active cell balancing of a 12cell lithium iron phosphate (LiFePO4) battery power system for an electric vehicle (EV). It is vital as energy storage system is present in all autonomous transport systems. The strong tracking cubature extended Kalman filter (STCEKF) gave an accurate SOC prediction compared to other Kalmanbased filter algorithms. The proposed GroupWise balancing of the multiple SOC exhibited a higher balancing speed and lower balancing loss than other cell balancing designs for EV under varying the ambient temperature. In summary, it is imperative that we continue to progress in our search for better intelligent autonomous transport systems design and simulation. The progress reported in this special issue suggests that achieving these aims is an unattainable one.

\author{
Cheng Siong Chin \\ Xionghu Zhong \\ Mohammad Hamdan \\ Rongxin Cui \\ Juan C. Cano \\ Jose R. Martinez-de Dios
}




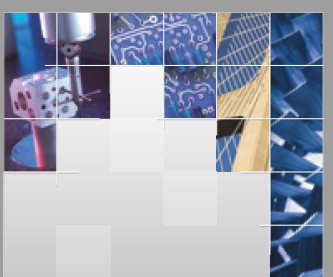

\section{Enfincering}
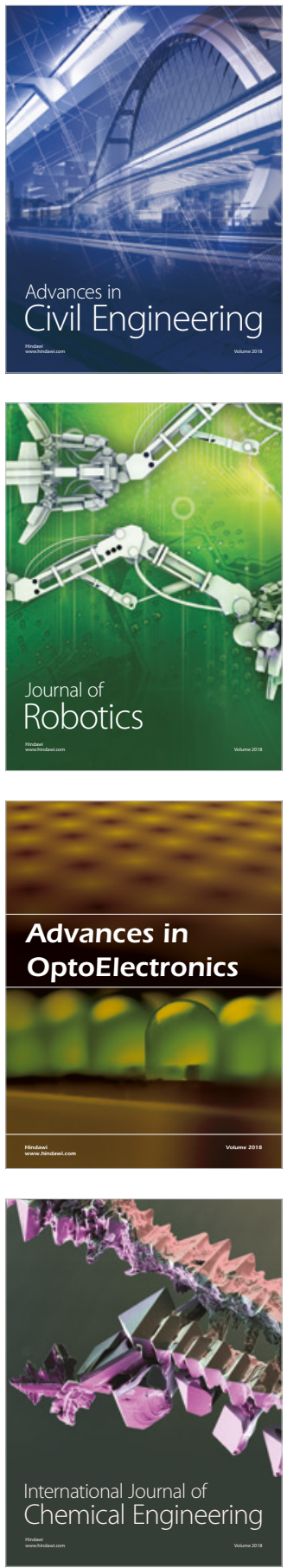

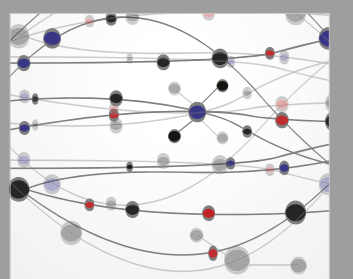

\section{Rotating \\ Machinery}

The Scientific World Journal

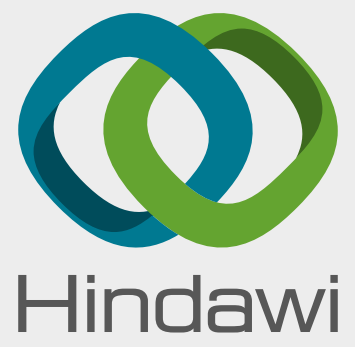

Submit your manuscripts at

www.hindawi.com
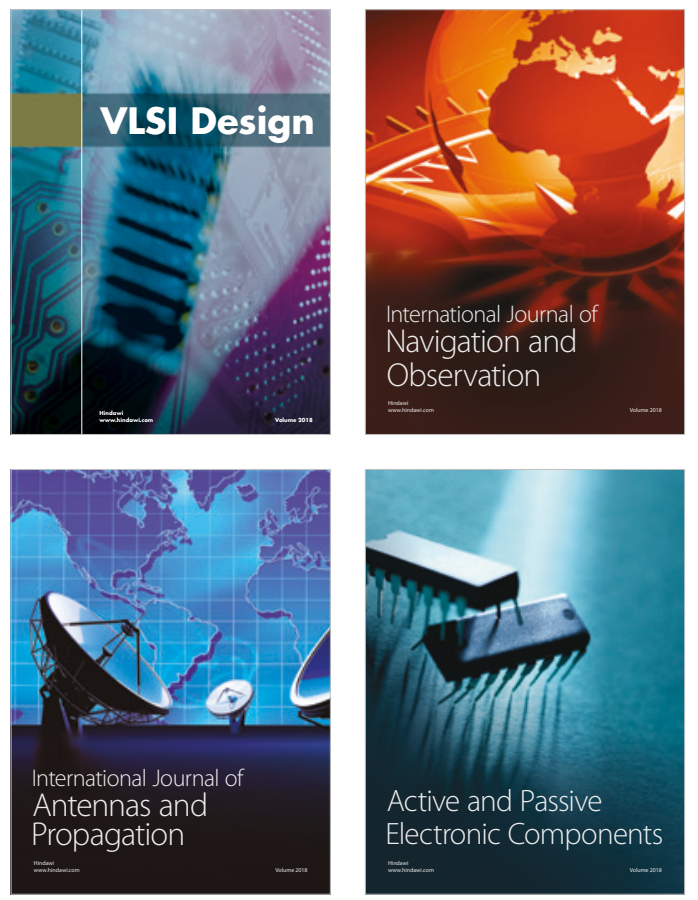
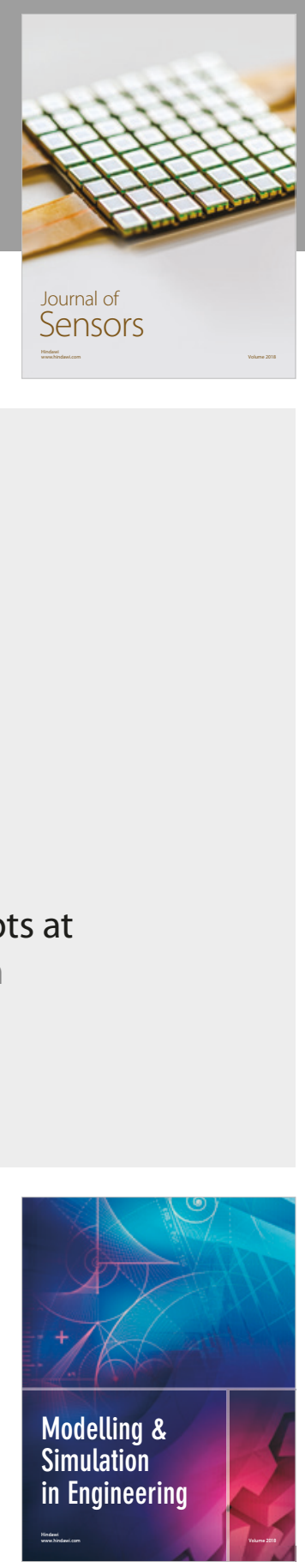

\section{Advances \\ Multimedia}
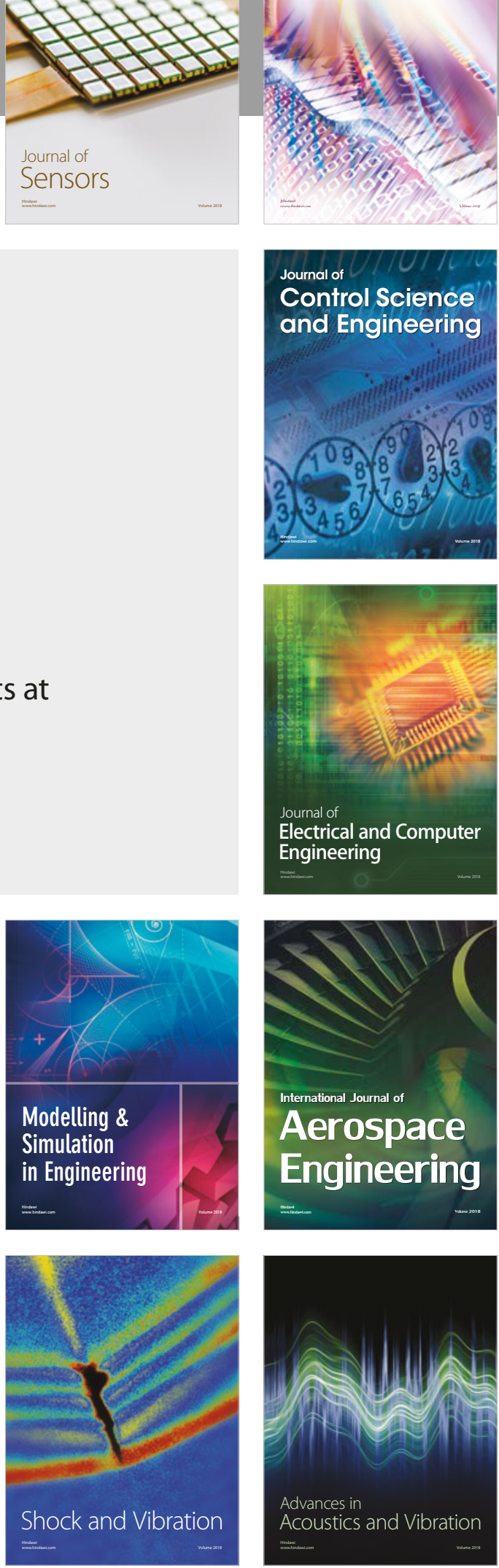